\title{
Early signs of critical slowing down in heart surface electrograms of ventricular fibrillation victims.
}

\author{
Berend Nannes $^{1}$, Rick Quax ${ }^{1,2[0000-0002-0299-0074]}$, Hiroshi \\ Ashikaga ${ }^{3[0000-0003-3689-6892]}$, Mélèze Hocini ${ }^{4,5,6[0000-0002-7957-8766]}$, Remi \\ Dubois $^{5,6[0000-0002-3320-6086]}$, Olivier Bernus ${ }^{5,6[0000-0003-3917-5791]}$, and \\ Michel Haïssaguerre ${ }^{4,5,6[0000-0001-7644-6146]}$ \\ 1 Computational Science Lab, University of Amsterdam Amsterdam, The \\ Netherlands \\ 2 Institute of Advanced Studies, University of Amsterdam, Amsterdam, The \\ Netherlands \\ 3 Cardiac Arrhythmia Service, Johns Hopkins University School of Medicine, \\ Maryland, USA \\ 4 Electrophysiology and Cardiac Stimulation, Bordeaux University Hospital, \\ Bordeaux, France \\ ${ }^{5}$ IHU LIRYC, Electrophysiology and Heart Modeling Institute, Bordeaux, France \\ 6 University of Bordeaux, Bordeaux, France
}

\begin{abstract}
Ventricular fibrillation (VF) is a dangerous type of cardiac arrhythmia which, without intervention, almost always results in sudden death. Implantable automatic defibrillators are among the most successful devices to prevent sudden death by automatically applying a shock to the heart when fibrillation occurs. However, the electric shock is very painful and could lead to dangerous situations when a patient is, for example, driving or biking. An early warning signal for VF could reduce the risk in such situations or, in the future, reduce the need for defibrillation altogether. Here, we test for the presence of critical slowing down (CSD), which has proven to be an early warning indicator for critical transitions in a range of different systems. CSD is characterized by a buildup of autocorrelation; we therefore study the residuals of heart surface electrocardiograms (ECGs) of patients that suffered VF to investigate if we can measure positive trends in autocorrelation. We consider several methods to extract these residuals from the original signals. For three out of four VF victims, we find a significant amount of positive autocorrelation trends in the residuals, which might be explained by CSD. We show that these positive trends may not be measurable from the original body surface ECGs, but only from certain areas around the heart surface. We argue that additional experimental studies involving heart surface ECG data of subjects that did not suffer VF are required to quantify the prediction accuracy of the promising results we get from the data of VF victims.
\end{abstract}

Keywords: Critical slowing down · Ventricular fibrillation · Critical transition · Early warning signal. 


\section{Introduction}

Ventricular Fibrillation (VF) is a dangerous type of cardiac arrhythmia where the ventricles, instead of beating normally, tremble uncontrollably. As a result, the heart is unable to regulate the blood circulation around the body, resulting almost always in sudden death. The most important contributions to the treatment of VF to date are automated defibrillators. For example, an implantable cardioverter-defibrillator (ICD) can be used for patients at an increased risk of suffering dangerous arrhythmia. The ICD detects cardiac arrhythmia and automatically intervenes by applying an electric shock. For these devices it is crucial to correctly detect the onset of $\mathrm{VF}$ in real time to be able to prevent permanent damage or death of the patient. In earlier research, several algorithms have been proposed to this end [1]. The ICD does not prevent the arrhythmia itself, but provides treatment by shock after the occurence. Intervention with ICD is not without drawbacks: the electric shock is experienced by patients as very painful [2] and can in some cases cause psychological problems [3]. Moreover, the shock could lead to dangerous situations if the patient is, for example, driving or biking. In the latter case, an early warning signal seconds before the shock could greatly reduce risk of accidents. Furthermore, early warning signals in combination with future methods to prevent VF from happening would reduce the need for defibrillation. Many possible indicators have been proposed in earlier work [4] from which Heart Rate Variability (HRV) is the most promising. However, contrary evidence has also been found, as HRV is influenced by characteristics - for example, medical conditions - of the individual patients [5]. Evidenty, a generic early warning signal for $\mathrm{VF}$ that is independent of patient characteristics is highly desirable.

If we look at VF from a general perspective, we can argue that the shift from a state where the ventricles are pumping normally to a state in which they quiver is a sudden transition between two different dynamical regimes for which the manifestation of VF is the tipping point. Such abrupt changes in dynamical behavior are seen in many real complex systems in nature and are generally called "critical transitions". In critical transitions, once the tipping point is exceeded, it is not easy to return to the previous state. A real-life example of this is desertification: once a patch of land reaches a barren state, it is hard for vegetation to reappear. This "irreversible" character has made possible predictors of these critical transitions much sought-after. Earlier research [6] has shown that there exists a domain-free early-warning signal for critical transitions in complex dynamical systems in different fields of research: critical slowing down (CSD). The theory behind CSD is based on the fact that, mathematically, some critical transitions in real systems can be interpreted as catastrophic bifurcations. It has been shown [7] that systems that approach such bifurcations experience a decrease in resilience; the system needs more time to recover from perturbations when a critical event is close. This decrease in resilience can be measured by increasing autocorrelation and standard deviation in the corresponding time series data (as we will explain in the Method section). These symptoms have indeed been identified in a wide range of real complex systems. For example: the ending 
of multiple different glacial periods by abrupt climate changes are preceded by the building up of autocorrelation in deuterium measurements [8], and brain activity shows increasing amounts of variance when close to an epileptic seizure [9]. Here, the same underlying principle applies, independent of the scientific field. If we assume that the onset of VF can be viewed as such a critical transition, we expect that we can detect the same early warning signals that are found in a variety of other real systems.

Historically, there has been controversy about the mechanism that drives VF [10], with some research suggesting that fibrillation represents a chaotic system [11], and others stating that it is rather similar to a nonchaotic random signal [12]. Nowadays it is commonly believed that the onset of VF is a transition to spatiotemporal chaos $[13,16]$ and thus a shift to a different, chaotic attractor. This transition is initiated by a wavebreak that arises when a wavefront and waveback of the cardiac excitation meet [15]. Under normal circumstances this never occurs, but in certain conditions the propagating impulse does not die out but returns to reexite the heart (called reentry [14]). When reentry triggers a wavebreak, it can in turn produce daughter waves, causing new wavebreaks etc., quickly degenerating into spatiotemporal chaos and VF. The transition from normal heart rhythm to abnormal, chaotic, heart rhythm (the process from reentry to VF) takes place through a series of bifurcations [16]. There exist different theories about how we can exactly describe this route to chaos [17]. In the context of nonlinear dynamics, however, we can consider the shift from a normal heart rhythm to VF as a change in topology of electrical wave dynamics or a transition between two states with different basins of attraction [18]. This drastic change in dynamical regime may therefore bear resemblance to critical transitions in other complex systems and may possibly be signaled by CSD.

In this thesis, we investigate if CSD can be observed in the residuals of heart surface electrocardiogram (ECG) recordings from patients that suffered VF. We analyze data sets from four patients provided by IHU LIRYC (Electrophysiology and Heart Modeling Institute) in Bordeaux, France. All patients suffered sudden cardiac arrest due to documented VF resulting from ischemic heart disease $(n=2)$, early repolarization syndrome $(n=1)$, or idiopathic VF $(n=1)$. All patients were male and the age range was between 15 and 74 years old. Each set contains around 1400 ECG signals (leads) over the heart surface, which are estimated using body surface ECG measurements by solving an ill-posed, inverse problem [19]. The original body surface potential maps consist of around 250 leads. Each lead in the set is a 20-second ECG recording; 10 seconds of normal heart rhythm followed by 10 seconds of arrhythmia, with a sampling rate of $1 \mathrm{kHz}$. We examine the 10 seconds of the signal that precede the tipping point looking for CSD. Specifically, we look for a significant increase in autocorrelation in the residual.

The main challenge of our research is the actual extraction of the residuals; the short-term fluctuations relative to the main ECG waves. To expose the residual, we have to filter out the wave components that are typical to the ECG. The difficulty resides in the high frequency characteristic of some of these typical 
waves, which impedes the use of a simple frequency filter. In this thesis, we go over several alternatives to correctly obtain the residuals, which is essential for credible autocorrelation measurements.

Electrocardiography is traditionally prone to different types of noise like power line interference, electrode contact noise and motion artifacts. We have to keep in mind that some of this noise (in particular, high frequency noise) we can not distinguish from fluctuations in the real signal and will end up in the residual. The ill-posed nature of the inverse solution may also induce errors in the residual. Therefore, to quantify the prediction accuracy of autocorrelation measurements for ECG data from VF victims, we have to conduct the same analysis on heart surface ECG estimations of subjects that did not suffer VF. The main goal of this thesis to show the necessity of such data.

\section{Method}

Critical slowing down (CSD) is the increase of recovery time needed by the system when it is perturbed from the stable state. In our case, this stable state is described by the well-known features of an ECG signal. Therefore, to be able to measure a possible CSD effect, we aim to find the fluctuations relative to the typical ECG curve: the residual. In this section we discuss several methods we considered to extract the residuals from the original signals and argue which method is the most preferable. To look for CSD, we have to analyze the time evolution of the autocorrelation of the extracted residuals. Because autocorrelation measures the likeness of a signal with a lagged version of itself, a slowly varying signal has a higher autocorrelation than a rapidly fluctuating signal. For that reason it is expected that, if residuals show a slowing down effect, we measure a significant positive trend in autocorrelation.

\subsection{Measuring the autocorrelation}

The most straightforward autocorrelation measure for equispaced data is the lag-1 autocorrelation, where the state of the signal at time $t$ is directly compared to its state in the previous time unit $t-1$. The lag- 1 autocorrelation can be estimated by treating the signal as a first-order autoregressive $(\operatorname{AR}(1))$ process and calculating the corresponding lag-1 autoregression coefficient. We estimate this parameter using Yule-Walker equations with Python library statsmodels.tsa.stattools. To capture the time evolution of the autocorrelation, the autoregression coefficient is calculated over a moving window. The window length is chosen to be exactly half the signal length, so that there is a reasonable trade-off between a sufficiently long window to compute the autocorrelation, and a long enough sequence of autocorrelation values to be able to study its time-evolution. (The influence of different window lengths on our result is shown in Appendix A.10) 


\section{$2.2 \quad$ Extracting fluctuations}

The typical ECG signal is composed of a set of main wave components (PQRST) formed by electrical currents produced by the depolarization and re-polarization of different heart chambers. Depolarization is responsible for the contraction of cardiac muscle, while with re-polarization, cardiac muscle relaxes. A schematic representation is provided in Fig. 1. The $\mathrm{P}$-wave is produced by the depolarization and contraction of both atria. The QRS-complex is composed of the electrical signals from both the depolarization of the ventricles and the re-polarization of the atria. Finally, the re-polarization of the ventricles produces the T-wave. The orientation of the waves is dependent on the polarity (positive or negative) of the electrode.

Normally, an effective technique to extract short term fluctuations from a signal would be to filter out the lower frequency components using by applying a high-pass filter. However, the QRS-complex has a significantly higher frequency range than the $\mathrm{P}$ - and $\mathrm{T}$-waves. A high-pass filter that removes all characteristic waves requires a high cutoff frequency, and thus imposes risk of filtering out short-term fluctuations that possibly show a CSD effect. The same problem arises for methods using wavelet decomposition: by removing high frequency subbands from the signal to remove the QRS complex we might accidentally remove fluctuations we want to analyze. For this reason, we have to consider other methods to extract the residuals: by utilizing the knowledge of the recurring wave components we create a model for the signal containing all characteristic waves and subtract it from the original signal.

In the following subsections we go over techniques we considered to extract the residues of the ECG leads.

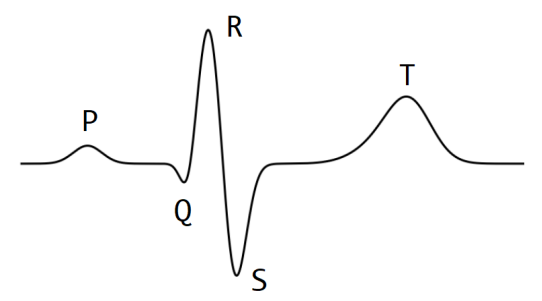

Fig. 1: The main wave components of an ECG; the P-wave, QRS-complex and T-wave.

Pre-processing We aim to remove characteristic components (Fig. 1) from the signal to extract the fluctuations. Baseline wandering in the signal makes it hard to distinguish wave components from the zero-volt level. Moreover, the height of characteristic waves may vary between heartbeats. In all methods discussed in this section, the baseline trend is first removed from the signal. Some methods 
require the signal to be cut into segments of one heartbeat cycle. These preprocessing steps are described in detail in Appendix A.1.

Fitting Gaussian curves To isolate the fluctuations in the ECG signal, we can directly subtract the characteristic ECG waves for every cardiac cycle. By fitting a mixture of Gaussian curves to the original ECG that approximate the PQRSTwaves we can construct an characteristic version (as shown in Fig. 1) of the signal, which can in turn be subtracted to reveal the residual. The implementation of this method is explained in more detail in Appendix A.2.

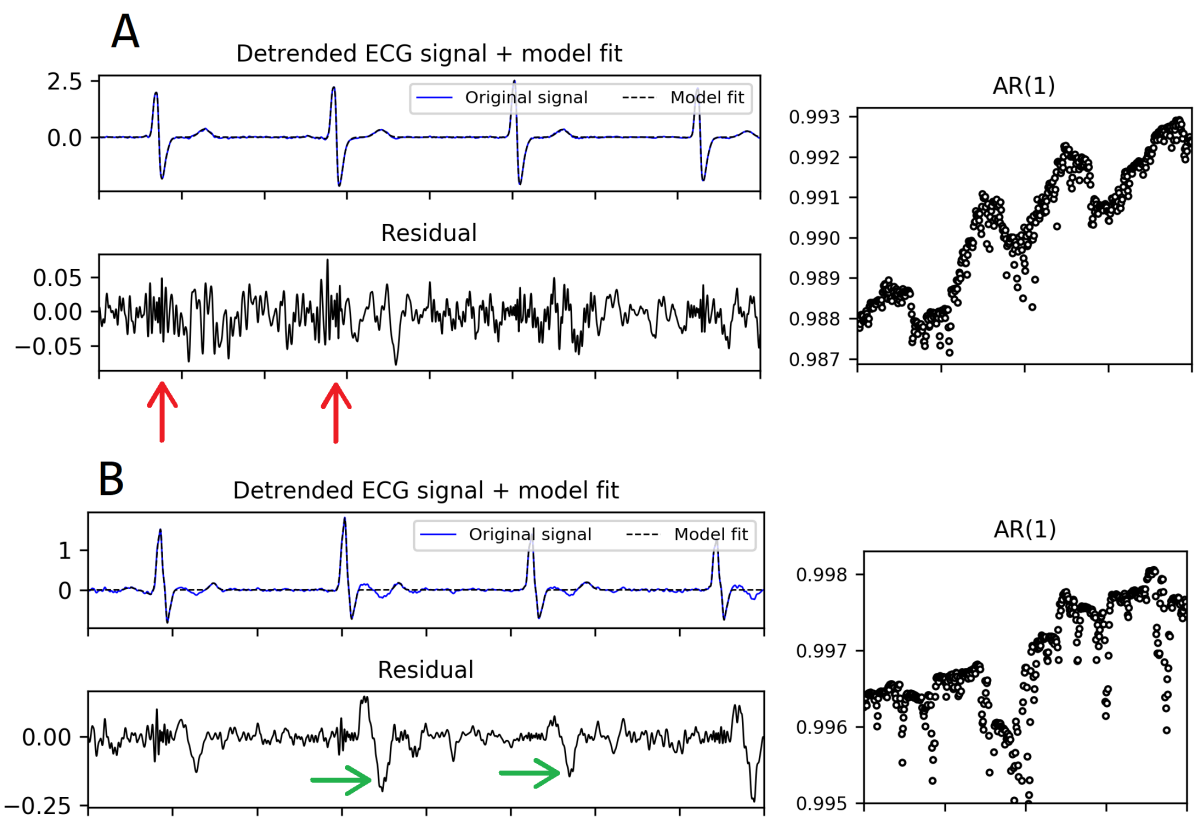

Fig. 2: Segments of detrended ECG signals, their corresponding model fit and the residuals after subtracting the model. A: The model seemingly fits the signal. However, we observe high frequency errors in the residual (red arrows) resulting in peaks and dips in the $\mathrm{AR}(1)$ measurements (right) occurring at the same frequency as the QRScomplexes. B: The signal, while we can identify the PQRST-waves, slightly deviates from the characteristic shape portrayed in Fig. 7. The fitting method fails to reproduce the characteristic waves resulting in periodically recurring errors in the residual (green arrows).

Techniques modeling ECG waveforms using Gaussian curves have been around for some time [21] and are generally used to extract clinical features like the location, height and width of the characteristic waves. When we apply our method to the ECG data we observe that indeed, for signals that resemble the PQRST- 
composition portrayed in Fig. 1 like the example given in Fig. 2A, the Gaussian fitting method seemingly provides a good model. However, when we subtract the model fit, the parts of the residual at the location of the QRS-complex clearly have higher frequency compared to other parts of the residual, which indicates that they are error artifacts induced by the fitting method. The effect of these errors is clearly visible in the $\mathrm{AR}(1)$ measurements: rapidly fluctuating parts of the signal have relatively low autocorrelation; combined with the moving window this results in peaks and dips in $\mathrm{AR}(1)$ values. These peaks and dips recur with the same frequency as the QRS-complexes. Clearly, while this modeling technique may be suitable for clinical feature extraction, it is not ideal to extract correct residuals of the signals. Moreover, to obtain a correct model, the signal has to resemble the characteristic shape from Fig. 1. When the signal deviates from this shape the fitting technique becomes infeasible, resulting in errors in the residual. An example of this is given in Fig. 2B. We conclude that this method is ineffective for the extraction of fluctuations from the signal since the majority of the signals in the provided data set do not have the required characteristic PQRST-composition.

Computing an average beat The method described above to extract fluctuations from the signal turns out to be ineffective as it requires each signal to have a certain characteristic shape. In reality, characteristics may be different for each signal. To capture characteristic features of a signal, one can also construct an average beat using the individual beat cycles. Characteristic features recur periodically and are thus automatically present in the average curve. Everything relative to the average beat can be classified as fluctuations. We cut the signal into segments containing one beat cycle. The fluctuations can be extracted beat by beat by subtracting the average beat from every cycle. However, the height as well as the overall shape of the characteristic waves is not constant over the whole signal. Therefore, the average curve must be adjusted for each beat to provide a good model for the signal. A detailed description of the average beat fit and these adjustments is given in Appendix A.3. It turns out that in almost all signals one or more QRS-complexes deviate too much from the average curve, even after the adjustments are made. As a result, the average curve is unable to fit the signal and errors occur in the residual (Fig. 3). For this reason, we conclude that the average beat method is unusable for credible autocorrelation measurements.

Excluding QRS-complexes In the methods discussed above, we encounter the problem that we can not (correctly) extract the fluctuations of parts of the signal around the QRS-complex. This inability is mainly caused by the fact that during the QRS-complex, a lot of change in y-axis value takes place in a limited amount of time points. Here, small fitting errors on the time axis can lead to big errors in y-axis value in the residual, and this heavily influences autocorrelation calculations. Therefore, to get more reliable results, it might be preferable to remove the QRS-complexes, or, generally, all parts with high first- 

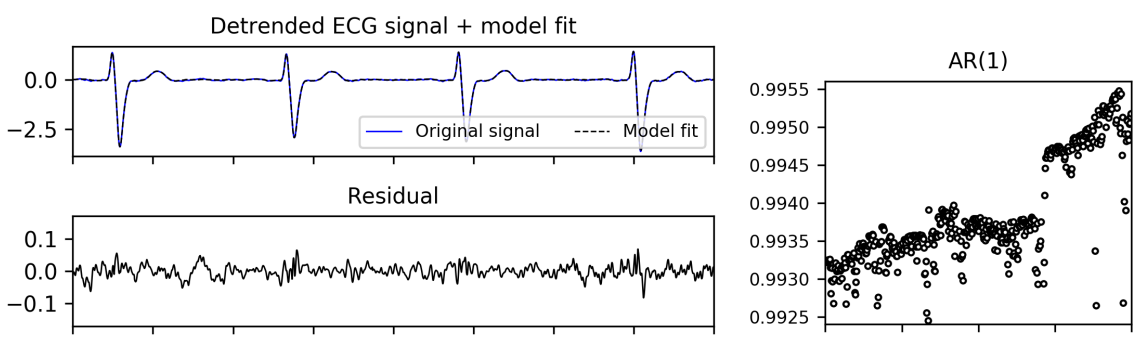

Fig. 3: Segment of an ECG signal and the average beat fit. The average beat is not able to fit all QRS-complexes, leading to jumps in the AR(1) measurements similar to Fig. 2A. This is typical for almost all signals in the data sets.

order differences, from the signal altogether. This has a clear disadvantage; we discard information by cutting parts of the signal. However, if the autocorrelation in the residual is gradually building up, this effect should still be observable, even if the signal is not complete. We implement a sequence of steps to remove unwanted parts of the ECG. This procedure accounts for the fact that, when cutting out parts of the signal, the difference in $y$-value at the edges might induce sudden "jumps" in the signal which affect any autocorrelation measurements. The cutting process is illustrated in Appendix A.4. Besides the fact that this procedure solves the problems we encountered in the methods mentioned above, it also opens up the possibility to use a frequency filter to extract the residual. As mentioned in the introduction of section 2.2, this filtering technique was not possible before due to the high-frequency characteristic of the QRS-complexes. Now that we are excluding these parts of the signal, we are able to extract the fluctuations using a simple low-pass filter. This is shown in Fig. 4: after removing the QRS-complexes the resulting signal is filtered using a $10 \mathrm{~Hz}$ cutoff frequency, which is sufficient to filter out any recurring ECG features. With this method, we are able to extract the residues of almost all signals without the major errors we encountered using other methods. We keep in mind that while the cutting procedure avoids major jumps in the resulting residual for most leads, it might still cause unwanted disruptions for some (for example, very noisy) signals. However, we have no reason to assume that this would result in more positive than negative trends in autocorrelation.

\section{Results}

We measure the trend of the lag-1 autocorrelation in the residuals calculated using the filtering method in 2.2. The parameter setting can be found in Appendix A.6. The trend is obtained by fitting a linear function to the calculated AR(1)-coefficients using a least-squares method and taking the slope. Evidently, applying this method to signals that exhibit CSD should result in significant positive slopes.

To determine the statistical significance of the slope of the AR(1) coefficients, we generate a distribution of $\mathrm{AR}(1)$-slopes from 1000 surrogate time series. The 

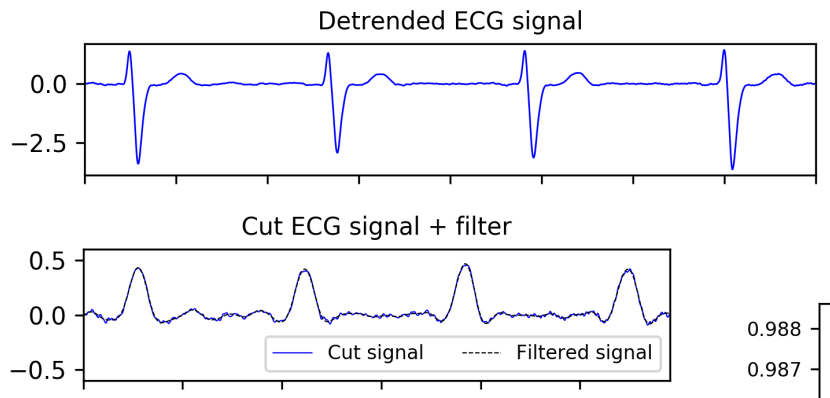

Residual
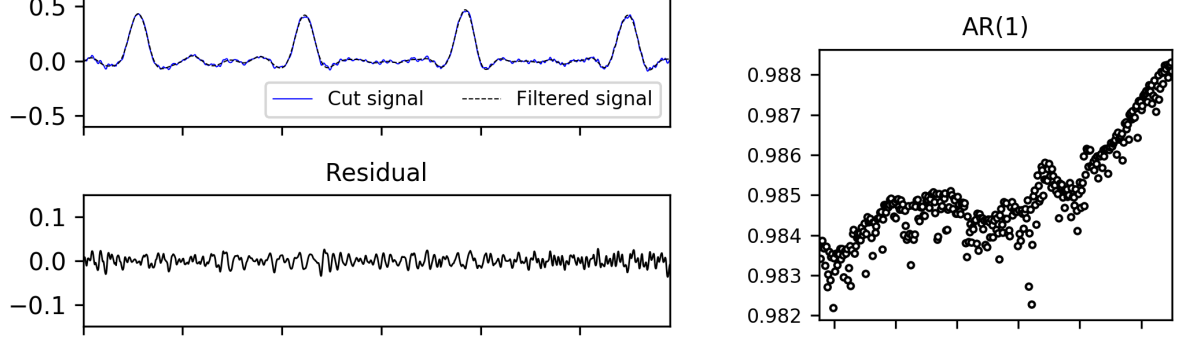

Fig. 4: ECG signal from Fig. 9 before (top) and after (middle) applying the cutting procedure in Fig. 13. The residual (bottom) is calculated by subtracting a filtered version of the signal (dotted line). A low-pass filter with a cutoff frequency of $10 \mathrm{~Hz}$ is used. The relative change in width of the plots represents the portion of the signal that is cut. The resulting $\mathrm{AR}(1)$ measurements form a smooth curve compared to the results from methods discussed above.

surrogate time series are created by taking the Fourier transform of the residual, multiplying the computed coefficients by random phases and transforming back. In the transformation, linear properties (amplitudes) are preserved and nonlinear properties (phase angles) are randomized. This way, the power spectrum is preserved and the surrogate time series have the same overall autocorrelation as the original residual but are random otherwise [22]. The AR(1)-slopes of the surrogates are normally distributed. A slope is considered significant if it does not fall within the two-sided $95 \%$ confidence interval of the obtained distribution. An example of this significance test is given in Appendix A.5.

Our null hypothesis is a situation where the heart is not close to VF, and no CSD is found in the corresponding ECG data. In that case, given the significance testing method described above, we should find an equal amount of positive and negative significant trends. We represent this by letting significant positive and negative slopes be drawn from a binomial distribution with success probability 0.5. $H_{0}: p=0.5$. We reject this null hypothesis if $p>0.5$ with a significance level of $5 \%$ (i.e. if the probability of $p=0.5$ is lower than $5 \%$ ). For cases where the null hypothesis is rejected the alternative hypothesis $H_{a}: p>0.5$ is accepted and are considered to have a substantial amount of significant positive trends that may possibly be explained by CSD.

We evaluate the trend of the autocorrelation in the ECG data of four patients that suffered VF. Each data set contains around 1400 leads. In Fig. 5 the slope of the AR(1) coefficients is plotted against the power (root mean square) of the residual. Each scatter plot represents results from a different patient. Significant 
positive or negative $\mathrm{AR}(1)$-slopes are highlighted in red. The figure shows that in three of the four cases there is a substantial majority of leads that show a significant positive trend in the lag-1 autocorrelation, compared to the number of significant negative trends. For these cases, $H_{0}$ is rejected and CSD might be at play. The corresponding scatter plots seem to have a similar shape, with the number of positive trends increasing as the power of the residual decreases. A possible explanation for this could be that residuals with high power contain a bigger proportion of measurement noise of the ECG recording, distorting the component of the residual that could contain CSD.
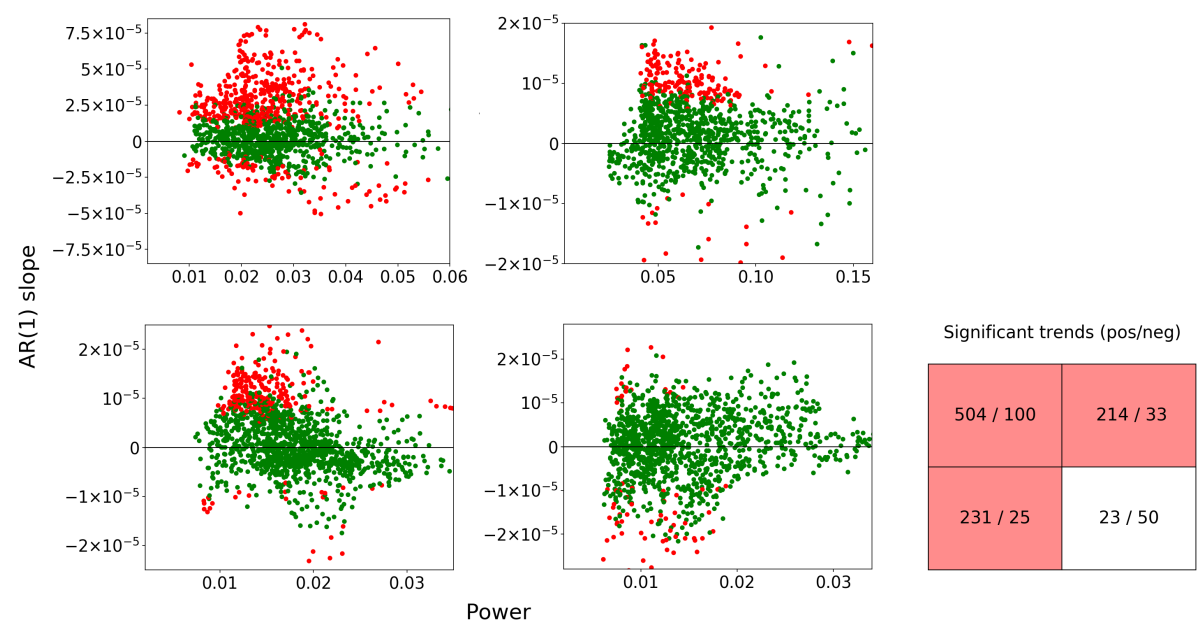

Fig. 5: Trend of the lag-1 autocorrelation in ECG residuals of four different VF events, plotted against the power of the residual. The trend is measured by the slope. Each dot represents one lead. Significant slopes are colored red. The table on the right shows the amount of significant positive/negative trends for the corresponding plot. For three out of the four patients $H_{0}$ is rejected (red cells) (Note that some points may be out of bounds for the sake of better visualization)

Before we draw the conclusion that the number of significant positive slopes is in fact the result of a CSD effect we examine sets of test data that consist of ECG signals from hearts that are not close to a VF event. Because we do not have access to similar heart surface electrograms for this category we use open source ECG data from PhysioNet [23] for this purpose. We analyze 9 sets of test data consisting of 10-second samples from 24-hour, $250 \mathrm{~Hz}$ Holter ECG recordings. As expected, we find no substantial majority of significant positive trends in any of these test sets. The results of the analysis of the test data are shown in Appendix A.7.

We also perform our analysis on the data set containing the original body surface ECGs that are used to compute the inverse solution. Strikingly, we do not observe the same substantial majority of positive trends we see for three of 
the four data sets of estimated heart surface ECGs. If our measurements are in fact the result of CSD, it seems this is not measurable with the original data and that the transformation to the inverse solution does provide extra information necessary to observe this effect.

An overview of all AR(1)-trend measurements is given in Appendix A.11. Given our significance testing method, under the null hypothesis (no CSD) we expect that around $5 \%$ of the autocorrelation trends we observe will be significant. Remarkably, for most data sets where $H_{0}$ is valid, we find that more than $5 \%$ of the trends are significant. It is likely that a portion of these significant trends is the result of residuals that are corrupted by noise, either directly (the noise directly influences the autocorrelation), or indirectly (the noise forces errors in the extraction of the residual, which in turn influences the autocorrelation). This can also be deducted from the plots in Fig. 15, where we can see that significant trends are relatively common for residuals with high power compared to points in the "main cluster". These results indicate that, while artifacts can cause more significant trends than expected, they occur both in positive or negative form, and are therefore not likely to lead to a rejection of $H_{0}$.

The sets of heart surface ECGs include triangulation coordinates, mapping each ECG signal to a point in 3D-space that corresponds to the location around the heart for which the inverse solution is calculated. We use this information to reproduce the plots from Fig. 5 where every point is color-coded based on its coordinates. These plots are shown in Appendix A.9. The results show that the points are clustered, which means that the significant positive trends in the heart surface data (red in Fig. 5) can be measured from specific angles, rather than all around the heart surface. We do not have enough information to couple the 3D-coordinates to a physical location of the heart surface; this would not be difficult to realize in future data acquisition. If the substantial amounts of significant trends are the result of CSD this can be valuable information, since one would know exactly where to look for possible early warning signals.

If, in further research, we can prove the presence of CSD in heart surface electrograms of VF victims, it may be possible for implantable devices such as ICDs to detect this effect and provide early warning signal for an oncoming arrhythmia. If, additionally, we can isolate from which area around the heart surface it can be measured, this may even be done by using just a single lead rather than the full potential map of the heart surface we used for this experiment.

\section{Conclusion and Disussion}

CSD has been used as a generic early warning signal for critical transition in a wide range of systems ranging from finance to climate. We reason that the heart as a complex system may bear similarities to such systems, since, in the context of dynamical systems theory, the transition from a normal heart rhythm to VF can be understood as a shift between two states with a different attractor. We hypothesized that when the heart is close to VF (i.e., close to the basin of attraction of the chaotic attractor) it may show decreasing resilience to pertur- 
bations, which can be measured as CSD. To test this hypothesis we investigate heart surface ECG signals right before the onset of VF.

In our results we indeed find signs of CSD: for three out of four VF victims, we find a substantial majority of significant positive autocorrelation trends in the residuals of heart surface ECG signals compared to the amount of significant negative trends. The heart surface ECGs are estimated by solving an inverse problem using body surface ECGs. If we perform the same analysis on the original body surface data we do not find such a majority, suggesting that, if CSD is in fact present, we have to compute the inverse solution to observe this effect. Furthermore, triangulation coordinates of the heart surface ECGs suggest that the possible CSD effect can only be measured from specific, yet unspecified angles surrounding the heart. We compare the results of the heart surface ECGs of $\mathrm{VF}$ victims to results from Holter recordings of subjects that are not close to a VF event. For the latter (no VF) data, we find that none of the nine recordings we analyze contain a substantial amount of significant positive autocorrelation trends and thus exhibit no CSD.

It has become clear, however, that this test data does not serve as an appropriate comparison the VF data for a number of reasons. Firstly, the test signals have a lower sampling rate of $250 \mathrm{~Hz}$ compared to the $1000 \mathrm{~Hz}$ of the VF data. It is possible that fluctuations in the residuals that show CSD can be captured by a sampling rate of $1000 \mathrm{~Hz}$ but not by a sampling rate of $250 \mathrm{~Hz}$. If this is in fact the case, using a sampling rate $250 \mathrm{~Hz}$ can not prove the absence of CSD in the test data. Secondly, with Holter recordings, the electrodes are placed on the chest of the patient. If we assume that the substantial amount of significant positive trends in the heart surface data is caused by CSD, our analysis of the data used to compute the inverse solution already seems to indicate that this effect is not as easily measured on the body surface. It would therefore only be logical if the test also does not show CSD. Lastly, the triangulation coordinates of the heart surface ECGs suggest that, if the data shows CSD, it can probably only be measured from specific angles surrounding the heart surface. Since the test data consists Holter recordings with only one lead, and thus only one angle of incidence, it is already unlikely to measure CSD for these signals.

The test data we use serves more as a validation of our method, rather than a validation of our result. While we do not expect CSD, the test signals are prone to the same types of noise as the VF data [25], which could influence autocorrelation measurements. Our analysis has shown, however, that this does not lead to a substantial majority of significant positive trends in any of the test data sets. This could indicate that the signs of CSD we find for the VF data are no artifacts of measurement noise. On the other hand, we can argue that some types of measurement noise (for example, noise caused by the movement of a patient) could affect multiple leads at once for the heart surface data since each lead covers that same ten-second time span. For this reason, the test data is still not sufficient to rule out the possibility that measurement noise influences our results. 
To prove that the predominant amount of significant positive autocorrelation trends we find in the residuals of heart surface ECGs of patients that suffered VF is in fact the result of CSD, we have to directly compare it to data of subjects that did not suffer VF, recorded in a similar manner. We therefore advocate further data acquisition. An important first step would be to obtain the 1400-lead heart surface ECG of subjects that did not suffer VF by solving the inverse problem using the body surface potentials. If the analysis of such data does not result in a majority of significant positive trends, this would be a strong indication that our measurements are showing CSD. Additional data from patients that suffered VF would also enable better statistical grounding. For future ECG recordings it is important to be able to map each signal to an exact physical location so that, if we can indeed prove CSD, we can also pinpoint angles from which this effect is measurable. So far we have checked for CSD by looking for a buildup in autocorrelation in the 10 seconds prior to the VF event. However, it is possible that this buildup initiates at an earlier time. If we can indeed use CSD as an early warning signal in this setting, it would be valuable to detect this as early as possible. For future data sets, it might therefore be useful to record ECG even longer before the onset of VF, where possible.

We concluded that for the extraction of the residuals from the signals it is best practice to remove parts of the signal with high first-order differences, which are otherwise hard to filter out. Evidently, for future research it is desirable to develop a method that can correctly extract residuals for the full signal, for example by improving the average beat method proposed in 2.2 or developing more advanced modeling techniques than the Gaussian fitting method in 2.2.

Acknowledgements This work was partly supported by the Fondation Leducq Transatlantic Network of Excellence 16CVD02.

\section{A Appendix}

\section{A.1 Pre-processing}

Removing baseline wandering The majority of the provided leads exhibit a baseline trend. Baseline wandering of the ECG signal might be caused by different phenomena like respiration or movement of the patient. Many different methods have been proposed the remove this baseline wandering [26]. We construct a filter that estimates the baseline drift, after which this drift is subtracted from the original signal to leave an ECG signal centered around the zero-volt level. The filter consists of a median filter with a moving window and a thirdorder Savitzky-Golay smoothing filter. The window lengths of both the median filter and the smoothing filter are set to a number of points corresponding to a $250 \mathrm{~ms}$ interval (250 points for a sampling rate of $1000 \mathrm{~Hz}$ ). The window lengths are chosen such that the intervals between the characteristic waves (the baseline) are aligned with the $\mathrm{x}$-axis. In some leads this may cause suppression of the T- 
and/or P-wave (which, in all proposed methods, are removed anyway in later filtering steps). This does not influence our measurements concerning fluctuations in the signal. The detrending process is illustrated in Fig. 6.

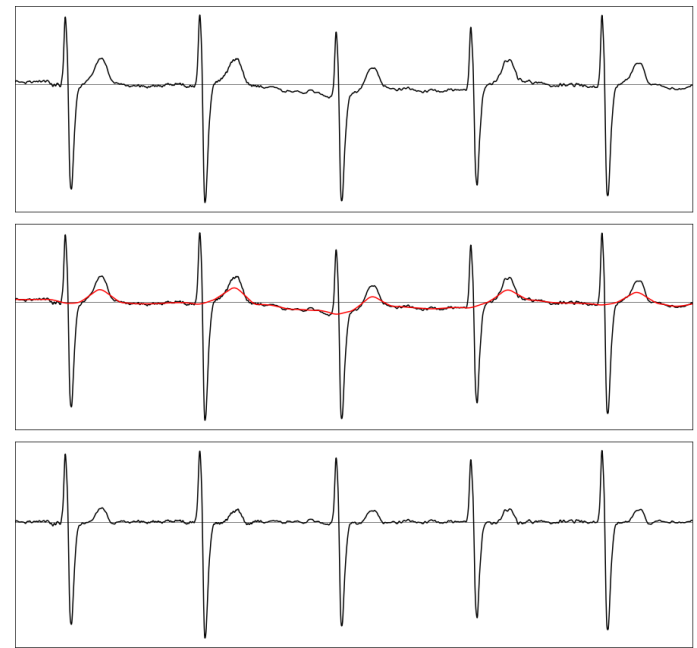

Fig. 6: top: original ECG signal. middle: ECG signal with estimated baseline drift (in red). bottom: ECG signal after removing baseline drift. (Note that the height of the T-wave is not preserved after filtering. This will not influence our results; we are interested in the preservation of short-term fluctuations rather than typical ECG components.)

Segmentation of the signal As explained in the opening of the Method section, we aim to create a model for the well known ECG components that we can subtract from the signal to reveal the residual. However, the height and/or shape of these components may vary from beat to beat. For some methods we will discuss in this section, it is therefore necessary to approach each beat separately. To do so, we divide the signal into segments, each containing one heartbeat cycle. We choose our segments to be centered around the R-peak; we cut the signal at every halfway point of the R-R interval. The locations of the R-peaks are detected by a simple peak-finding algorithm that finds the biggest local maxima with a minimum distance between them. Depending on the quality of the ECG data, other methods can be used to this end; for example, advanced QRS-detection algorithms [27].

\section{A.2 Gaussian curve fitting method}

In 2.2 we propose a method to extract fluctuations from an ECG signal by subtracting a model version of the signal composed of a mixture of Gaussians. In 
this model, each component except the T-wave is described by a single Gaussian curve of the form $2 a_{i} \exp \left(-\left(t-t_{i}\right)^{2} / 2 w_{i}^{2}\right)$. Here $a_{i}$ represents the amplitude of the curve, $w_{i}$ is the width, and $t_{i}$ is the position on the time-axis. For the Twave, two Gaussian curves are used to account for its asymmetry. The ECG signal is cut into heartbeat segments as described in A.1. The model curve is then composed of the sum of six Gaussians, each described by three variables :

$$
f(t, \mathbf{a}, \mathbf{w}, \mathbf{t})=\sum_{i} 2 a_{i} \exp \left(-\left(t-t_{i}\right)^{2} / 2 w_{i}^{2}\right) \quad i \in\left\{P, Q, R, S, T_{1}, T_{2}\right\}
$$

The composition of the ECG model is illustrated in Fig. 7

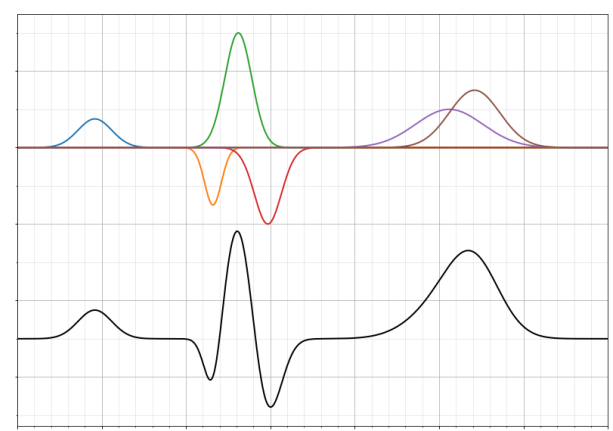

Fig. 7: Composition of the model ECG curve (bottom): six Gaussian curves (top) represent the characteristic ECG components.

The optimal fit is found by solving the 18-variable nonlinear least-squares problem minimizing the cost function:

$$
\epsilon=\|s(t)-f(t, \mathbf{a}, \mathbf{w}, \mathbf{t})\|^{2}
$$

Where $s(t)$ is the original ECG signal. Good estimates for $a_{R}, a_{S}, t_{R}$ and $t_{S}$ can be found by peak-finding methods (like described in Appendix A.1), and are set as initial values to reduce the run time of the least-squares procedure. Initial guesses for $t_{i}$ and $a_{i}$ values for other waves can be chosen relative to $t_{R}$ and $a_{R}$ using general knowledge about normal ECG intervals lengths and relative signal strengths. Similarly, initial estimates for $w_{i}$ can be made using typical wave durations. Furthermore, boundaries are set for the search space of the least-squares algorithm to maintain the orientation and order of the PQRSTwaves.

\section{A.3 Average beat fit method}

In this section, we describe the method proposed in 2.2 to compose an average beat, which can be subtracted from the signal in order to extract the residual.

First, we cut the signal into segments containing one beat cycle. The individual segments are aligned by locating the $\mathrm{R}$ - and $\mathrm{S}$ - peaks and finding the 
intersection with the y-axis in between. This is chosen as point of alignment because it is the part of the signal with the highest first-order derivative and therefore the most prone to errors in the y-direction due to placement errors on the $\mathrm{x}$-axis. The average beat is then computed by taking the mean of all the individual beat cycles. The fluctuations can be extracted beat by beat by subtracting the average beat from every cycle. As mentioned earlier, the height of the characteristic waves is not constant over the whole signal. Therefore the average curve must be adjusted to match the signal strength of the respective beats. We do so by calculating a multiplication factor for all points in the time series by dividing the value of the signal by the value of the average beat. For points with amplitude below a certain (small, for example $<0.1$ ) threshold, the multiplication factor is set to one (to avoid division by very small numbers).
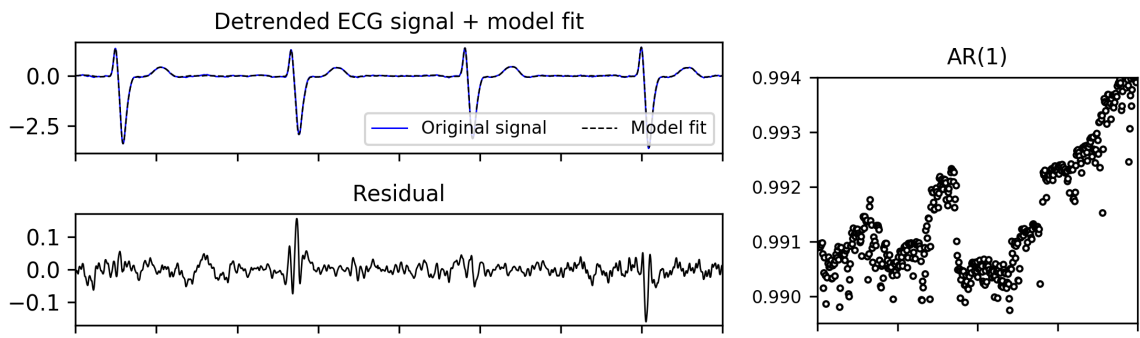

Fig. 8: Segment of an ECG signal, the average beat fit and the resulting residual after subtraction. While the height of the waves is matched by the average beat fit, errors appear in the residual as a result of the varying shape of the QRS-complexes. We observe dips and peaks in the $\mathrm{AR}(1)$ measurements similar to the errors we encountered with the previous method.
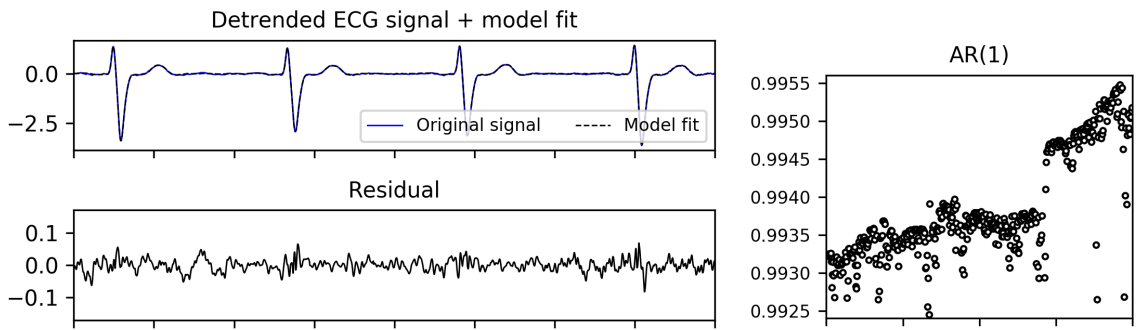

Fig. 9: ECG signal from Fig. 8 and the model fit after applying the correction method. While the errors seen in the residuals in Fig. 8 are somewhat suppressed, jumps in the AR(1) measurements is still clearly visible. This is typical for almost all signals in the data sets.

Then the average beat is multiplied by a smoothed version of the multiplication factor array, so that it matches the original signal amplitude, but does not 

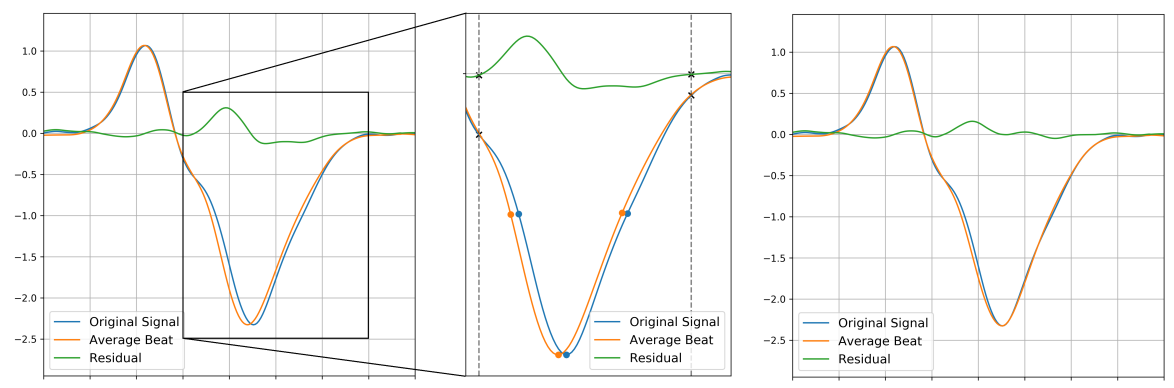

Fig. 10: left: Original ECG signal, average beat and residual. The S-wave in this segment is slightly lagged compared to the average, resulting in a large error in the residual. middle: For each wave in the QRS-complex, the location of the peak and the two halfway points are compared for the original signal and the average beat. The wave is considered to be lagged if at least two of these three points occur at a later time. If so, two fixed points (where the residual is zero) are chosen to on the left and right side of the wave. For both the original and the average curve, the length of the segment from the left fixed point to the peak and the segment from the peak to the right fixed point are compared. Next, both the segments of the average curve are resampled to match the length of the segments from the original signal. right: Original signal, average beat and residual after applying the correction technique.

contain any short term fluctuations from the signal. In most cases, the smoothed multiplication curve is unable to reproduce the $\mathrm{Q}, \mathrm{R}$ and $\mathrm{S}$ peak heights, because the QRS-complex is characterized by higher frequencies. To correct this, an additional multiplication curve is constructed for the QRS-complex by calculating the multiplication factor at the peaks, setting the multiplication factor to one at the intersections with the y-axis, and interpolating using a linear spline for the remaining points.

While this technique accounts for the varying heights of the QRS-waves, errors in the residual still appear due to variations in the overall shape of the QRS-complex (see Fig. 8). We introduce two adjustment steps in an attempt to limit such errors in the residual, which are described in Fig. 10 and Fig. 11. The adjustment steps use resampling of parts of the signal to optimize the fit of the average curve. While this procedure improves the quality of the fit in most cases, often one or more of the QRS-complexes deviate too much from the average curve for the correction method to succeed. In Fig. 9 we show what the residual from Fig. 8 looks like after applying the correction.

We conclude that this correction method is not sufficient to remove all errors in the residuals. An example in which this method is unable to remove the error is given in Fig. 12.

\section{A.4 Excluding QRS-complexes}

In 2.2 we propose a method to remove parts of the signal with high first-order derivative since they often lead to errors in the residual. When cutting out parts 

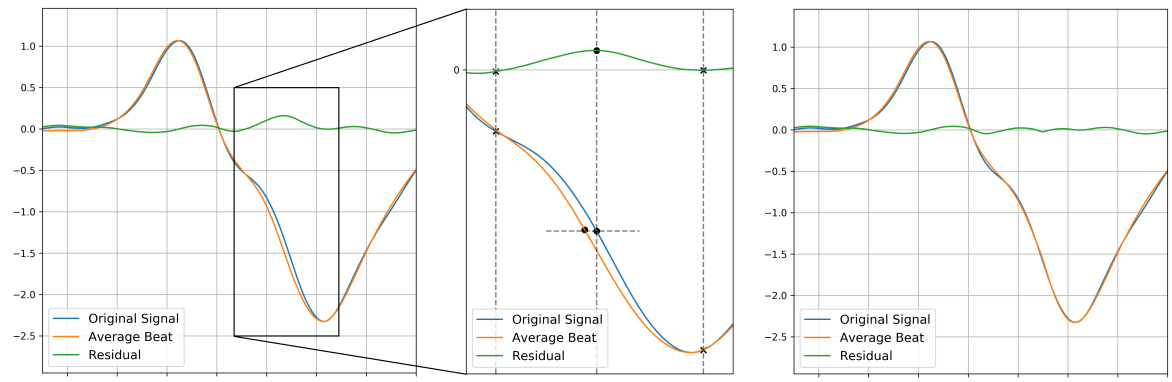

Fig. 11: left: Original signal ECG, average beat and residual after applying the correction step from Fig. 10. middle: Two fixed points (where the residual is zero) are chosen at the base of the error in the residual. Then, the central point on the original signal and the corresponding point (in y-axis value) on the average beat are found and the displacement is calculated. Next, the segment of the average curve from the left fixed point to the central point and the segment from the central point to the right fixed point are resampled to match the segment lengths of the original curve. right: Original signal, average beat and residual after applying the correction technique.
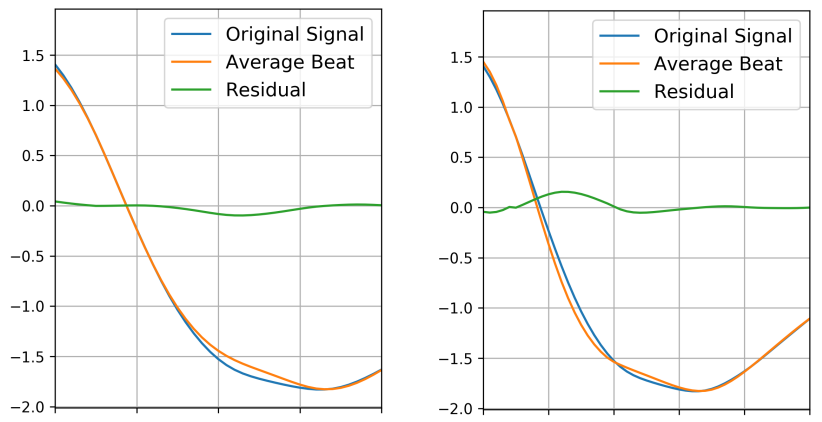

Fig. 12: Segment of an ECG signal (S-wave) before (left) and after (right) applying the correction method described in Fig. 11. The shape of the wave deviates from the average wave in such a way that the method can not correct this. On the contrary, in this case, the error in the residue increases by applying the correction method.

of the signal, one has to take into account that sudden jumps may appear in the resulting signal. In Fig. 13 we introduce a method that avoids these jumps for most signals.

\section{A.5 Significance testing}

We measure the trend in the lag-1 autocorrelation of the residuals by fitting a line to the calculated $\mathrm{AR}(1)$-coefficients and taking the slope. To determine the significance of the autocorrelation trend of a residual, we compare it to the autocorrelation trends of 1000 surrogate residuals. The surrogates are created as follows: First, we take the Fourier transform of the original residual and randomize the phase angles. The amplitudes of the Fourier components are kept. Then 

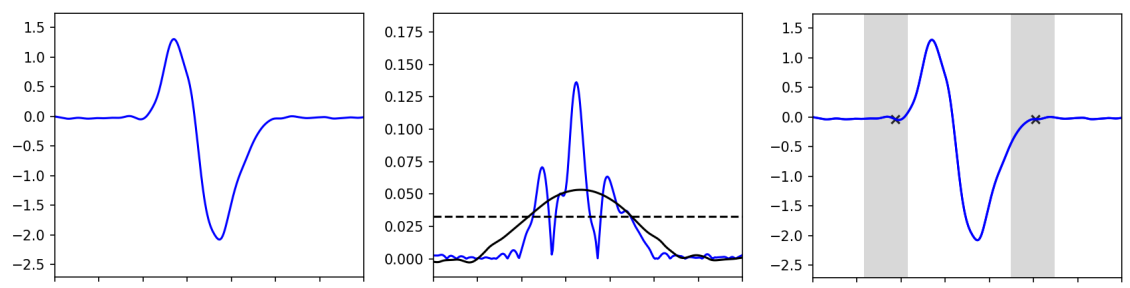

Fig. 13: Cutting procedure for ECG signals. left: Original signal. middle: First, the first-order differences of the signal are calculated (blue line). As initial guesses, the intersections of a smoothed version of the first-order differences (black line) with a threshold (dotted) line (of a number of standard deviations, depending on the noisiness of the data) are taken. right: A search space of a fixed number of points to the left and right of the initial guesses is defined (grey area). Within these search spaces, the two points with the least difference in $\mathrm{y}$-axis value are chosen as cutting points (black crosses)

the surrogate signal is obtained using the inverse Fourier transform. By construction, the original and the surrogate signal have the same power spectrum and overall autocorrelation [22]. However, the time evolution of the autocorrelation is different for both signals. An example is given in Figure 14, where a residual (A) and one of its surrogate signals (B) are shown. The autocorrelation trends of the surrogates are normally distributed; Figure 14 shows the distribution of AR(1)-slopes of 1000 surrogates of residual (A). The slope is considered significant if it falls outside the $95 \%$ confidence range (i.e. more than 1.96 standard deviations from the average) of the distribution. The range for which slopes are significant is colored red. In this example, the original residual is considered to have a significant positive autocorrelation trend.

\section{A.6 Parameter settings}

To extract the residuals from the data leading to the results in Section 3, we use the following settings in our cutting procedure:

The first-order differences of the signal are smoothed using a Savitzky-Golay filter with a moving window of 3 points.

As initial cutting points, the intersections of this smoothed version with a threshold line are taken. The threshold must be chosen by hand for each data set according to the noisiness of the data. This can easily be done with only a few trial signals. The value of the threshold usually lies between $0.75-2$ standard deviations.

Next, the width of the search space is chosen relative to the average R-R interval; in our case, 1/20 part of the average number of points $\mathrm{R}-\mathrm{R}$ interval. Choosing the search space relative to the beat intervals is best practice, as it may vary between patients. This is also the right way to account for switching between different sampling rates. 

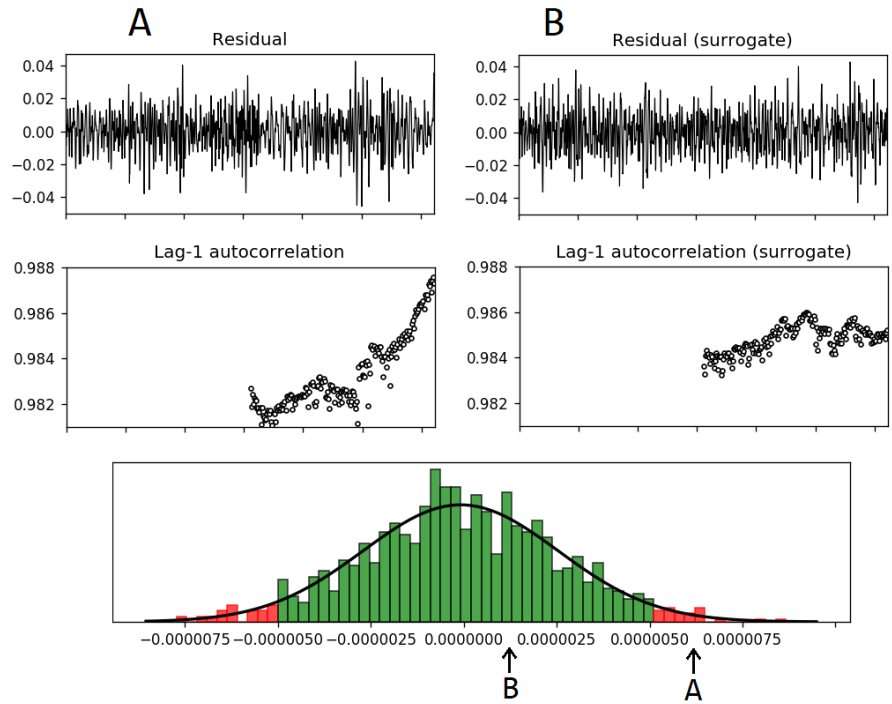

Fig. 14: top: Residual (A), one of its surrogate signals (B). middle: The corresponding $\mathrm{AR}(1)$ measurements. bottom: Distribution of AR(1)-slopes of 1000 surrogates of residual (A). The value of the slope of (A) and (B) are indicated with an arrow.

After the unwanted parts of the signal are cut out, we apply a low-pass filter of $10 \mathrm{~Hz}$ to the signal. The filtered version is then subtracted from the signal to obtain the residual.

\section{A.7 Test data}

To validate our analysis methods, we examine sets of test data that consist of ECG signals from hearts that are not close to a VF event. We take 10-second samples from 24-hour, $250 \mathrm{~Hz}$ Holter ECG recordings of patients that eventually suffered VF. We make sure, however, that the samples are taken hours before the actual VF event and we expect no slowing down effect to take place. We use Holter recordings from the database [24] with sinus (normal) rhythm and with no known history of disease or medication. We choose to take 1400 samples per subject, which is approximately the amount of leads in each heart surface data set.

We analyze the test sets using the same approach as before, taking 10-second samples, computing the trend in autocorrelation and plotting against the power. The results of 2 of the 9 test sets are shown in Fig. 15. From the plots, we can immediately observe that the number of positive and negative trends is much more balanced, as they look almost symmetrical about the x-axis. This is also true for the significant trends; we see approximately equal amounts of significant positive and negative trends, and $H_{0}$ is not rejected. The results of all 9 test data sets are included in Appendix A.11. 

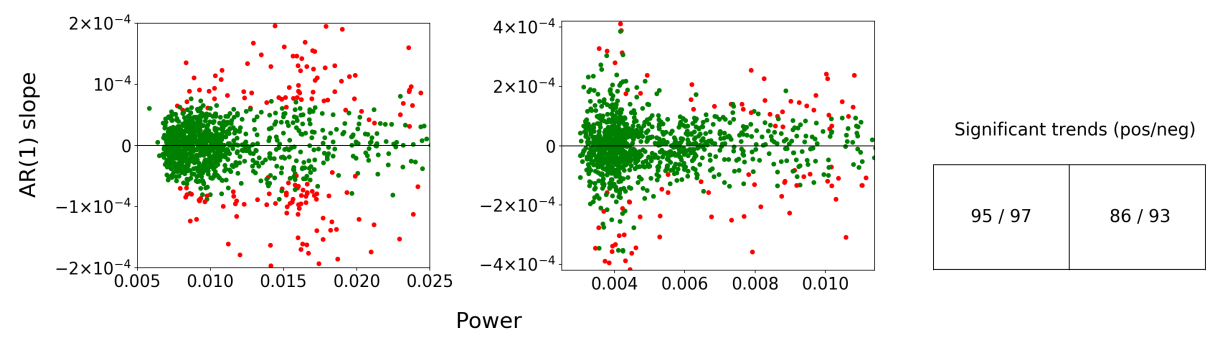

Fig. 15: Trend of the lag-1 autocorrelation in ECG residuals from test samples from Holter recordings of two different individuals plotted against the power of the residual. The trend is measured by the slope. Each dot represents one 10-second sample taken from the recording. Significant slopes are colored red. The table on the right shows the amount of significant positive/negative trends for the corresponding plot. For these cases $H_{0}$ is not rejected. (Note that some points may be out of bounds for the sake of better visualization)

\section{A.8 Body surface ECGs}

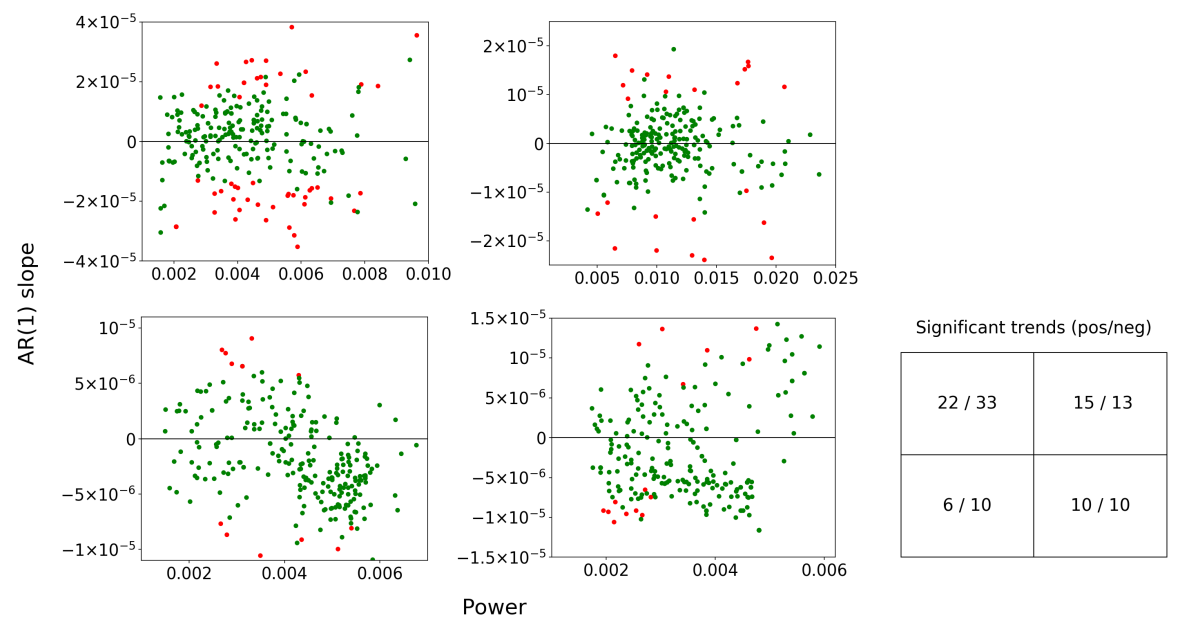

Fig. 16: Trend of the lag-1 autocorrelation of the body surface ECGs that are used to estimate the heart surface leads we analyze (Figure 5). The trend, measured as the slope of a linear function fitted to the data, is plotted against the power of the residual. The table on the right shows the amount of significant positive/negative trends for the corresponding plot. (Note that some points may be out of bounds for the sake of better visualization)

In Figure 5 we show autocorrelation trend measurements for heart surface ECG residues of patients that suffered VF. The heart surface ECGs are estimated using body surface ECG measurements by solving an ill-posed, inverse problem. 
We perform the same analysis on these original signals to investigate if we observe a similar majority of significant positive trends we see for the estimated heart surface leads. The resulting plots are shown in Figure 16. The individual plots are linked to the plots in Figure 5 (the top-right plot contains the body surface data from the top-right plot in Figure 5, etc.). Evidently, in these plots, no notable majority of positive trends is visible. This indicates that, if the majority of significant positive trends in the heart surface data is the result of CSD, this effect might not be measurable in the corresponding body surface data. (See Table 1 for a complete overview of the measurement results.)

\section{A.9 Triangulation}

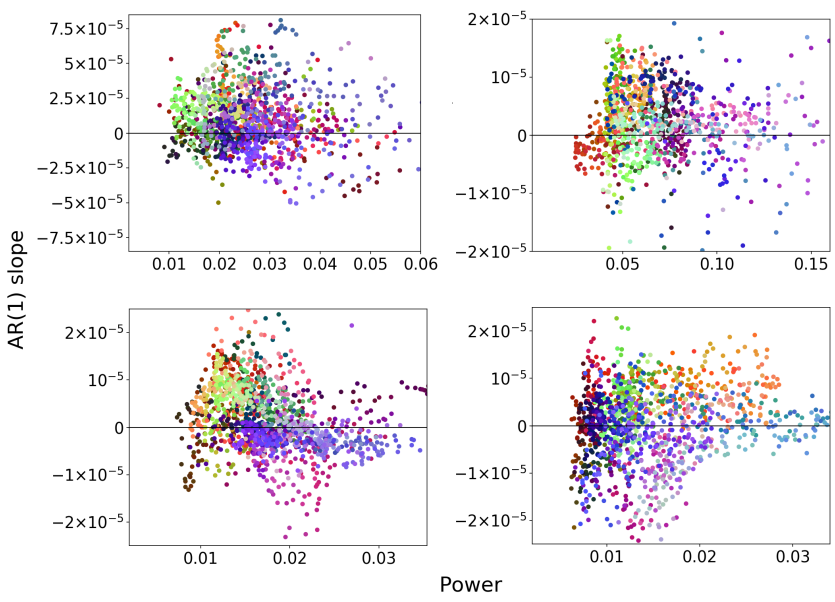

Fig. 17: Trend of the lag-1 autocorrelation in ECG residuals of four different VF events, plotted against the power of the residual. The plots contain the same measurement results as Figure 5. The color of the dots (RGB-value) is based on the (x,y,z) coordinates of the location around the heart for which the inverse solution is calculated, by $(\mathrm{r}, \mathrm{g}, \mathrm{b})$ $=(\mathrm{x}, \mathrm{y}, \mathrm{z})$. The triangulation of the data sets differ from each other, meaning that dots with the same color in different plots do not necessarily have the same physical location.

The estimated heart surface ECGs we analyze are labeled with triangulation coordinates which correspond the point in 3D-space for which the inverse solution is calculated. We use this information to reproduce Figure 5, but with colorcoded points based on the 3D-coordinates: Figure 17. From the plots we can see that the points are clustered. This indicates that the substantial amount of significant trends we observed for this data can be measured from specific angles. If the positive trends are in fact the result of CSD, it would be valuable if we can link these angles to physical locations on the heart surface so we know where to look for early warning signals. The triangulation differs between the individual 
plots, meaning, for example, that yellow points in one plot do not necessarily originate from the same position as yellow points in another plot, etc. In future data acquisition, it is therefore useful to keep track of the physical mapping of the data points.

\section{A.10 Window lengths}

The length of the moving window over which the autocorrelation of the signal is measured is important for the results of our analysis. Our results are obtained by using a window size of 0.5 times the signal length. We illustrate the effect of different window sizes by showing results of our autocorrelation trend measurements for window sizes of $0.25,0.5$ and 0.75 times the signal length (Figure 18). The window length must be sufficiently long for a realistic measurement of the
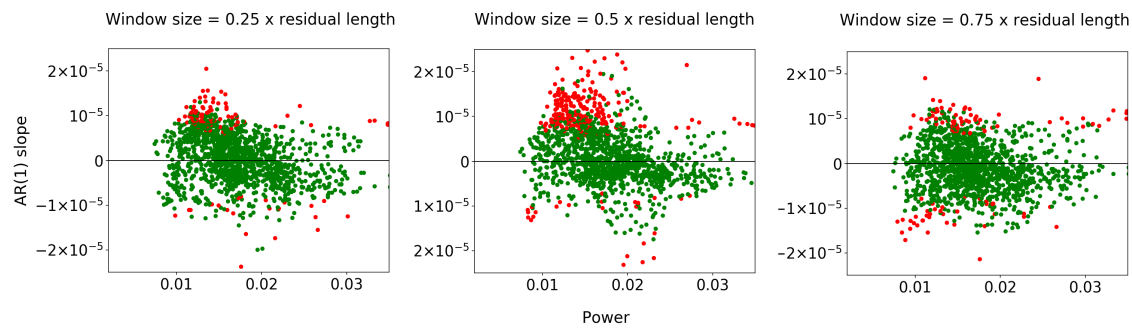

Fig. 18: Trend of autocorrelation against the power of the residual for three different autocorrelation window sizes: $0.25,0.5$ and 0.75 times the signal length, respectively.

autocorrelation of the signal; short window lengths allow more fluctuation in the trend, since the autocorrelation is calculated over a small number of points. Figure 18 shows that this results in a smaller amount of positive significant trends, while the majority of the significant trends are still positive. On the other hand, we require a long enough sequence of autocorrelation values to be able to study its time-evolution; if we take a large window size, we are only able to capture the time evolution of a small part of the signal, which is more likely to deviate from the overall trend. This is also visible in Figure 18 we observe that choosing a large window size results in a more balanced number of positive and negative trends. Taking this into account, we argue that taking the window length to be exactly half the signal length is the best trade-off.

\section{A.11 Summary results}

This section contains an overview of the autocorrelation measurements for ECG data of VF victims (over the heart surface and body surface) and Holter test recordings of subjects that are not close to VF. The number of significant positive and negative trends found for each data set is shown in Table 1. 
Table 1: Number of significant positive and negative trends in the lag-1 autocorrelation of ECG residuals in the VF data sets, containing 10-second signals right before the onset of $\mathrm{VF}$, and in the test data sets, containing 10-second samples of Holter recordings hours before the onset of VF. Cases where $H_{0}$ is rejected are colored red and are considered to have a substantial majority of significant positive trends.

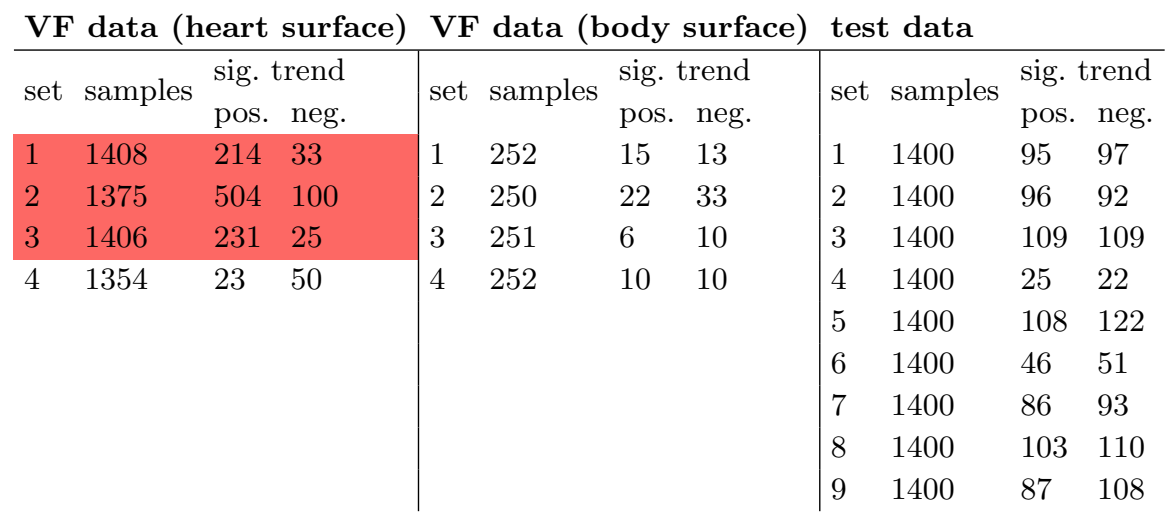

\section{References}

1. Amann, A., Tratnig, K., Unterkofler, K.: Reliability of old and new ventricular fibrillation detection algorithms for automated external defibrillators. Biomedizinische Technik/Biomedical Engineering. 48, 216-217 (2003).

2. Ahmad M., Bloomstein L.Z., Roelke M., Bernstein A.D., Parsonnet V.: Patients' attitudes toward implanted defibrillator shocks. Pacing and clinical electrophysiology 23(6), 934-938 (2000)

3. Sears S.F., Conti J.B.: Quality of life and psychological functioning of ICD patients. Heart 87(5), 488-493 (2002)

4. Lane, R.: Prediction and prevention of sudden cardiac death in heart failure. Heart. 91(5), 674-680 (2005).

5. Reed, M., Robertson, C., Addison, P.: Heart rate variability measurements and the prediction of ventricular arrhythmias. QJM. 98(2), 87-95 (2005).

6. Scheffer, M., Bascompte, J., Brock, W., Brovkin, V., Carpenter, S., Dakos, V., Held, H., van Nes, E., Rietkerk, M., Sugihara, G.: Early-warning signals for critical transitions. Nature. 461(7260), 53-59 (2009).

7. Wissel, C.: A universal law of the characteristic return time near thresholds. Oecologia. 65(1), 101-107 (1984).

8. Scheffer, M., Dakos, V., Nes, E., Brovkin, V., Petoukhov, V., Held, H.: Slowing down as an early warning signal for abrupt climate change. Proceedings of the National Academy of Sciences. 105(38), 14308-14312 (2008).

9. McSharry, P., Smith, L., Tarassenko, L.: Prediction of epileptic seizures: are nonlinear methods relevant?. Nature Medicine. 9(3), 241-242 (2003).

10. Goldberger, A., Bhargava, V., West, B., Mandell, A.: Some observations on the question: Is ventricular fibrillation "chaos"?. Physica D: Nonlinear Phenomena. 19(2), 282-289 (1986).

11. Surawicz, B.: Ventricular fibrillation. The American Journal of Cardiology. 28(3), 268-287 (1971). 
12. Kaplan, D., Cohen, R.: Is fibrillation chaos?. Circulation Research. 67(4), 886-892 (1990).

13. Garfinkel, A., Chen, P., Walter, D., Karagueuzian, H., Kogan, B., Evans, S., Karpoukhin, M., Hwang, C., Uchida, T., Gotoh, M., Nwasokwa, O., Sager, P., Weiss, J.: Quasiperiodicity and chaos in cardiac fibrillation. Journal of Clinical Investigation. 99(2), 305-314 (1997).

14. Wit, A., Cranefield, P.: Reentrant excitation as a cause of cardiac arrhythmias. American Journal of Physiology-Heart and Circulatory Physiology. 235(1), H1H17 (1978).

15. Weiss, J., Qu, Z., Chen, P., Lin, S., Karagueuzian, H., Hayashi, H., Garfinkel, A., Karma, A.: The Dynamics of Cardiac Fibrillation. Circulation. 112(8), 1232-1240 (2005).

16. Weiss, J., Garfinkel, A., Karagueuzian, H., Qu, Z., Chen, P.: Chaos and the Transition to Ventricular Fibrillation. Circulation. 99(21), 2819-2826 (1999).

17. Qu, Z.: Chaos in the genesis and maintenance of cardiac arrhythmias. Progress in Biophysics and Molecular Biology. 105(3), 247-257 (2011).

18. Qu, Z., Hu, G., Garfinkel, A., Weiss, J.: Nonlinear and stochastic dynamics in the heart. Physics Reports. 543(2), 61-162 (2014).

19. Gulrajani, R.: The forward and inverse problems of electrocardiography. IEEE Engineering in Medicine and Biology Magazine. 17(5), 84-101, 122 (1998).

20. Clifford, G., McSharry, P.: Method to filter ecgs and evaluate clinical parameter distortion using realistic ECG model parameter fitting. Computers in Cardiology. pp. 715-718. IEEE, Lyon (2005).

21. Suppappola, S., Sun, Y., Chiaramida, S.: Gaussian pulse decomposition: An intuitive model of electrocardiogram waveforms. Annals of Biomedical Engineering. 25(2), 252-260 (1997).

22. Schreiber, T., Schmitz, A.: Surrogate time series. Physica D: Nonlinear Phenomena. 142(3-4), 346-382 (2000).

23. Goldberger, A., Amaral, L., Glass, L., Hausdorff, J., Ivanov, P., Mark, R., Mietus, J., Moody, G., Peng, C., Stanley, H.: PhysioBank, PhysioToolkit, and PhysioNet. Circulation. 101(23) (2000).

24. Greenwald S.D.: Development and analysis of a ventricular fibrillation detector. M.S. thesis, MIT Dept. of Electrical Engineering and Computer Science, 1986. https://alpha.physionet.org/content/sddb/1.0.0/

25. Everss-Villalba, E., Melgarejo-Meseguer, F., Blanco-Velasco, M., Gimeno-Blanes, F., Sala-Pla, S., Rojo-Álvarez, J., García-Alberola, A.: Noise Maps for Quantitative and Clinical Severity Towards Long-Term ECG Monitoring. Sensors. 17(11), 2448 (2017).

26. Jayant, A., Singh, T., Kaur, M.: Different Techniques to Remove Baseline Wander from ECG Signal. International Journal of Emerging Research in Management \& Technology. 2(6), 16-19 (2013).

27. Pan, J., Tompkins, W.: A Real-Time QRS Detection Algorithm. IEEE Transactions on Biomedical Engineering. 32(3), 230-236 (1985). 